\title{
Effect of intervention on the rates of breastfeeding of very low birth weight newborns
}

\author{
Walter Santoro Júnior, ${ }^{1}$ Francisco Eulógio Martinez ${ }^{2}$
}

\begin{abstract}
Objective: To describe an intervention to provide support and encouragement to mothers of preterm newborns and to evaluate its effect on breastfeeding rates in the first 6 months after hospital discharge.

Methods: One hundred newborns and their mothers were selected consecutively and prospectively according to order of birth. The mother-infant dyads were alternately assigned to one of two groups: one group received routine care (routine group), and the other group received the intervention (intervention group). The intervention consisted of individualized support in addition to the routine support provided in the Neonatology Service. Before delivery, one of the researchers was introduced to the mother and offered her information about the delivery. The researcher was also present at delivery, made an early visit to the infant in the intensive care unit, provided constant support for the mother to express breast milk and to maintain lactation, gave the mother information about maternal and infant hospital discharge, and provided outpatient follow-up after discharge.
\end{abstract}

Results: Thirty-six dyads completed the study. The characteristics of mothers and infants, as well as causes of exclusion, were similar in the two groups. In the routine group, $38.9 \%$ of the infants were being breastfed at hospital discharge, and median breastfeeding duration was 54 days. In the intervention group, $80.5 \%$ were being breastfed at discharge, and median breastfeeding duration was 91 days $(\mathrm{p}<0.01)$.

Conclusions: Simple support measures offered to mothers during hospitalization and outpatient follow-up had a very positive effect on breastfeeding rates.

J Pediatr (Rio J). 2007;83(6):541-546: Prematurity, breastfeeding, preterm newborn, feeding, nutrition, human milk.

\section{Introduction}

Establishing breastfeeding of preterm newborns is a great challenge. Several factors make it difficult, and the most evident are physiological limitations. Preterm newborns only achieve maturation to suck and swallow between the 32nd and 35th week postmenstrual age, which complicates the determination of an ideal time to initiate breastfeeding. ${ }^{1,2}$

In addition to the infant's immaturity, maternal factors may also make breastfeeding difficult. Early separation of mothers from infants is frequent because of the need to care for the infants in a neonatal intensive care unit. The postpartum period is a time of maximum maternal sensitivity, and mothers may fail to establish an affective bond with their infant at the most appropriate time because of the separation of the dyad. ${ }^{3}$ Moreover, mothers are worried about the separation from their infants and their survival and growth, and may feel incompetent to perform their role, which generates further anxiety. ${ }^{4,5}$ Studies in the literature emphasize that the creation of an affective bond between mother and infant and a low level of anxiety are important to establish breastfeeding. ${ }^{6-8}$

The difficulties to breastfeed a preterm infant continue after hospital discharge. Many infants being breastfed at discharge are weaned earlier than desired. ${ }^{9}$

Some studies describe programs to promote breastfeeding among mothers of preterm infants. ${ }^{10}$ They stress the

1. Doutor, Faculdade de Medicina de Ribeirão Preto, Universidade de São Paulo (USP), Ribeirão Preto, SP, Brazil.

2. Professor titular, Faculdade de Medicina de Ribeirão Preto, USP, Ribeirão Preto, SP, Brazil.

No conflicts of interest declared concerning the publication of this article.

Suggested citation: Santoro Júnior W, Martinez FE. Effect of intervention on the rates of breastfeeding of very low birth weight newborns. J Pediatr (Rio J). 2007;83(6):541-546.

Manuscript received May 29 2007, accepted for publication Sep 102007.

doi 10.2223/JPED.1724 
importance of providing support to mothers by qualified professionals, ${ }^{11-14}$ and the benefits of support offered by the community and the family. ${ }^{15-18}$

The purpose of this study was to describe a breastfeeding intervention to provide support and information to mothers of preterm newborns and to evaluate its effects on breastfeeding rates in the first 6 months after hospital discharge.

\section{Methods}

This study was conducted in the Neonatology Service of Hospital das Clínicas, School of Medicine of Ribeirão Preto, Universidade de São Paulo, from February to November 2001. Infants included in the study weighed less than $1,500 \mathrm{~g}$ at birth. Preterm newborns and their mothers were assigned to one of two groups: one group received routine care (routine group), and the other group received the intervention (intervention group). The groups were formed simultaneously, and allocation followed the consecutive and alternate order of birth of the infants. By drawing lots, it was determined that the first infant would be assigned to the routine group. A sample size of at least 27 infants in each group was calculated according to previous data about breastfeeding in the first week after discharge detected in our Service ${ }^{9,19}$ (about 40\%) and the expectation of an increase of $40 \%$ due to the intervention (to over $80 \%$ ) based on a two-tailed alpha value of 0.05 and beta value of 0.10 .

Exclusion criteria were all situations that prevented breastfeeding due to the infant's or the mother's conditions. Therefore, the following exclusion criteria were established: severe neurologic problems or facial malformation that made breast sucking difficult; digestive tract malformations; hospitalization for longer than 4 months; HIV+ mother; and death. Twins were included, but multiple births were excluded.

When the mother of a preterm infant was in labor, she received and explanation about the study and, in case she agreed to participate and signed an informed consent term, previous randomization was checked. If the infant belonged to the intervention group, the researcher was called and stayed with the mother in the labor room. In case the infant was assigned to the routine group, the researcher was only informed that a mother had been included in the routine group.

This study was approved by the Ethics in Research Committee of the Hospital de Clínicas de Ribeirão Preto, School of Medicine, Universidade de São Paulo, according to HCRP approval number 5899/2001.

The birth of a high-risk newborn is routinely assisted by a team whose basic concern is to support the infant's life. No healthcare worker is assigned to give the mother information about the infant's clinical conditions or treatment in the first hours of life.
Whenever possible, the preterm newborn should have physical contact with the mother in the delivery room. The mother's first visit to the infant in the intensive care unit is organized by the nursing team, but, due to the nurses' time availability, the mother may be separated from the infant for hours.

All puerperal mothers receive instructions about how to express milk, empty the breasts and increase liquid ingestion. Milk expression every 2-4 hours is suggested, and sterile containers are provided by the Milk Bank. Milk expression should begin as early as possible, and the duration of milk expression depends basically on the mother's well being.

During hospitalization, the mother should have unrestricted access to her infant, but many times she is discharged from the hospital earlier than her infant. After discharge, the mothers receive instructions and are encouraged to visit their infants without restrictions of time or duration.

When the infant is ready for hospital discharge, the pediatrician reinforces the importance of continuing breastfeeding. The mother receives instructions to offer the breast first, and to offer complements with artificial milk only when prescribed by the pediatrician. The first visit to the prematurity outpatient service is scheduled one week after hospital discharge.

This study's intervention began before delivery, when the researcher was formally introduced to the mother and answered her questions about delivery. At that time, it was made clear that the researcher would provide information about the infant's conditions to the mother but would not be directly involved in the infant's care. The purpose of the physical presence of the researcher was to offer support at a critical moment and to establish a relationship of trust with the patient.

The mother's visit to the infant in the intensive care unit was encouraged and occurred as early as possible to promote the creation of an affective bond between mother and infant. It took place as soon as the newborn was taken to the intensive care unit if the infant was clinically stable and the mother was ready to understand the information that she was going to receive. When a longer time was necessary to define the newborn's conditions, waiting time was up to 4 hours. When the mother's condition did not allow her to make an early visit, information was taken to her by the researcher while she was still in the post-anesthesia care unit. At the time of the visit, the mother received a simple description of what was going on, of the invasive or noninvasive monitoring system, of the infant's conditions, and of treatment in the next hours. Information was repeated to the mother as many times as necessary.

The initiation and continuation of the mother's milk production followed our Service's routine procedures. In the intervention group, however, the researcher individually reinforced 
previous instructions about the techniques to express milk, empty the breast, and increase liquid intake.

Mothers in the intervention group were encouraged to personalize their infant's crib or incubator. They could place identification labels where they wished, and were encouraged to bring small gifts that expressed their affection and their fast recovery wishes. Mothers other than those included in the study and who also chose to personalize their infants' cribs were not stopped by the healthcare team.

At the mother's hospital discharge, support was offered again and instructions about the continuation of milk production and treatment were repeated. The importance of visiting the infant, or making phone calls when visits were not possible, was also reinforced. The researcher was present during the visits whenever possible or when requested by the parents by phone. The parents' access to the unit was unrestricted.

When an infant of the intervention group was discharged, the researcher gave the parents a cell phone number for contact. The first visit was scheduled one week after discharge. However, depending on the case and when problems could not be solved over the phone, an extra visit was scheduled before that date.

Outpatient follow-up visits in both groups were monthly for 6 months after hospital discharge or until weaning. In the routine group, residents assisted by professors or other physicians provided care to the infants, but the infants in the intervention group were seen by the researcher. After leaving the intervention study, routine neonatology outpatient follow-up was ensured for the children not in the intervention group.

Data about the two groups were recorded and reviewed by the researcher; therefore, the study was not blinded. Exclusive breastfeeding was defined as feeding the infant with only the mother's milk, and breastfeeding was any situation in which the infant received the mother's milk, regardless of whether exclusively or not.

The Fisher exact or the chi-square test was used for the analysis of tables. The unpaired $t$ test was used for the comparison of normally distributed data, the Mann-Whitney test, for nonnormally distributed data. Kaplan-Meier survival curves were compared using a log rank test. The EpiInfo 6.02 software was used for statistical analysis of data, and the level of significance was set at $p<0.05$.

\section{Results}

In 10 months, 100 consecutive mothers of preterm newborns in the Neonatology Service of Hospital de Clínicas de Ribeirão Preto, Universidade de São Paulo, agreed to participate in the study. Fifty-one were assigned to the routine group, and 49 , to the intervention group. Thirty-six infants in each group completed the study, that is, 15 were excluded in the routine group and 13 in the intervention group. All infants participating in the study were followed up in the outpatient service and there was no loss to follow-up up to the sixth month after hospital discharge or up to weaning.

The causes of exclusion were similar in the two groups, and the main causes were prolonged hospitalization (32\%) and death (43\%).

The characteristics of mothers and newborns included in the study are shown in Table 1. Mothers and infants in the two groups had similar characteristics. Maternal age in both groups ranged from 14 to 43 years, and mean age was 26.1 years. Most mothers lived in the city of Ribeirão Preto $(61.1 \%)$, were multiparas (57\%), had previous experience in breastfeeding (54.2\%), studied up to the end of elementary school (69.4\%), and had a cesarean delivery (72.2\%).

The infants had a mean weight of $1200 \mathrm{~g}$ at birth and a mean gestational age of almost 32 weeks; about $60 \%$ had adequate weight for gestational age, and over $95 \%$ had good vital signs at 5 minutes of life. Mean hospitalization time was 47 days; at hospital discharge, median weight was $1955 \mathrm{~g}$ and mean postmenstrual age was 38.7 weeks.

The rate of weaning and no breastfeeding in the routine group at the time of hospital discharge was $61.1 \%$. This value was significantly higher than the $19.5 \%$ found in the intervention group. This value remained statistically different between groups at 3 months of outpatient follow-up. In the routine group, the rates of exclusive breastfeeding and breastfeeding were $8.4 \%$ and $38.9 \%$ at hospital discharge and $5.6 \%$ and $36.1 \%$ at the time of the first visit. In the intervention group, these rates were $19.5 \%$ and $80.5 \%$ at hospital discharge and $16.6 \%$ and $75 \%$ in the first visit. Half of the infants receiving artificial milk at hospital discharge (11 in the routine group and 3 in the intervention group) did not have access to the maternal breast at any moment in their lives. Median breastfeeding duration in the group of infants that were breastfed, excluding, therefore, those that were never breastfed, was 54 days in the routine group and 91 days in the intervention day $(p<0.001)$.

Figure 1 shows the Kaplan-Meier survival curves of continuation of breastfeeding according to study group. The intervention group had better breastfeeding indices during all the study, and the curves were statistically different $(p<0.01)$.

Only 4 infants in the routine group and 7 in the intervention group were being exclusively breastfed at hospital discharge. Figure 2 shows the Kaplan-Meier survival curve of breastfeeding for these 11 infants, from hospital discharge to the end of outpatient follow-up, compared with the 33 infants that were receiving nonexclusive breastfeeding at hospital discharge. The curves are statistically different $(p<0.01)$.

\section{Discussion}

The benefits of breastfeeding for preterm newborns are undeniable, but the rates of breastfeeding among these 
Table 1 - Characteristics of mothers and very low birth weight preterm newborns included in the study.

\begin{tabular}{|c|c|c|c|}
\hline & \multicolumn{3}{|c|}{ Study groups } \\
\hline & Routine group & Intervention group & $\mathbf{p}$ \\
\hline Maternal age (years)* & $24.5 \pm 7.7$ & $27.7 \pm 6.4$ & 0.06 \\
\hline Primipara, $\mathrm{n}(\%)$ & $16(44.4)$ & $15(41.6)$ & $1^{+}$ \\
\hline Experience in breastfeeding, $\mathrm{n}(\%)$ & $20(55.5)$ & $13(36.1)$ & $0.15^{+}$ \\
\hline Elementary school education, $\mathrm{n}(\%)$ & $29(80.5)$ & $21(58.3)$ & $0.07^{+}$ \\
\hline Secondary or college education, $\mathrm{n}(\%)$ & $7(19.4)$ & $15(41.6)$ & $0.07^{+}$ \\
\hline Birth weight $(\mathrm{g})^{*}$ & $1.193 \pm 219$ & $1.208 \pm 188$ & 0.76 \\
\hline Gestational age (weeks)* & $31.7 \pm 1.8$ & $31.9 \pm 1.9$ & 0.78 \\
\hline Small for gestational age, $\mathrm{n}(\%)$ & $13(36.1)$ & $16(44.4)$ & $0.63^{+}$ \\
\hline 5-minute Apgar $\geq 7 \mathrm{n}(\%)$ & $34(94.4)$ & $35(97.2)$ & $1^{+}$ \\
\hline SNAPPE-II ${ }^{\ddagger}$ & $5(0-34)$ & $12(0-38)$ & 0.67 \\
\hline Hospitalization (days)* & $49.4 \pm 17.2$ & $46.5 \pm 16.2$ & 0.47 \\
\hline Weight at discharge $(\mathrm{g})^{\ddagger}$ & $1.970(1.900-2.885)$ & $1.940(1.870-2.700)$ & 0.23 \\
\hline Postmenstrual age at discharge (weeks)* & $38.9 \pm 1.7$ & $38.5 \pm 2.2$ & 0.34 \\
\hline
\end{tabular}

SNAPPE-II = Score for Neonatal Acute Physiology Perinatal Extension II.

* Mean \pm standard deviation - unpaired $t$ test.

+ Fisher exact test.

* Median (range) Mann-Whitney test.

infants are still low. ${ }^{9,10}$ Therefore, methods to improve these rates should be studied for preterm newborns.

Few studies in the literature specifically investigate breastfeeding of very low birth weight infants. There are few intervention models to provide support to mothers and to

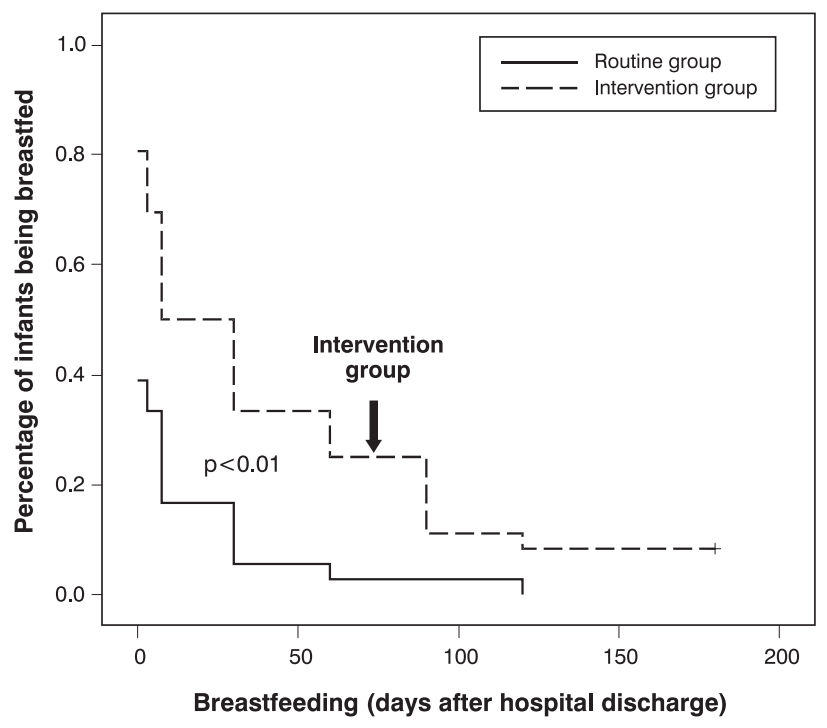

Solid line = routine group; dashed line = intervention group.

Figure 1 - Kaplan-Meier survival curve: breastfeeding according to study group

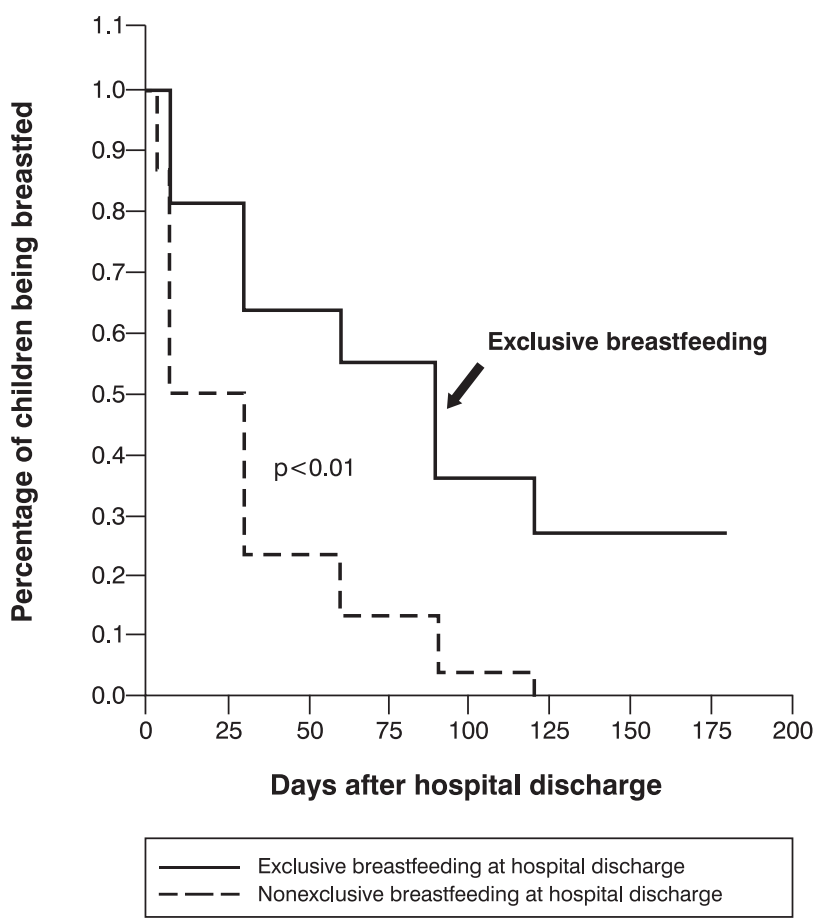

Solid line = exclusive breastfeeding at hospital discharge; dashed line = nonexclusive breastfeeding at hospital discharge.

Figure 2 - Kaplan-Meier survival curves of breastfeeding from hospital discharge to the end of outpatient follow-up for 11 infants exclusively breastfed at hospital discharge and for 33 infants that were receiving nonexclusive breastfeeding at hospital discharge 
encourage breastfeeding of preterm newborns, and few evaluations of the development of these infants. Moreover, study results are conflicting. Therefore, studies should investigate models of interventions to encourage and sustain breastfeeding of preterm newborns. ${ }^{10,20-24}$

This study evaluated the impact of a very simple intervention offered to the mothers of preterm newborns from the delivery room to outpatient follow-up. This intervention consisted of having the researcher close to the mother to offer support and information and to answer her questions.

Despite the significant rate of exclusion of mother-infant dyads recruited for the study, the sample size was large enough to detect the beneficial effects of this intervention. Randomization was adequate in that there were no differences in the characteristics of the dyads studied. The support offered had a very important effect on improving breastfeeding rates at hospital discharge.

At hospital discharge, $38.9 \%$ of the children in the routine group were being breastfed: $8.4 \%$ were exclusively breastfed, and $30.5 \%$, nonexclusively. These results are similar to previously described preterm newborn breastfeeding rates in our Service. ${ }^{16,17}$ In contrast, $80.5 \%$ of the children in the intervention group were being breastfed at hospital discharge: $19.5 \%$ were exclusively breastfed, and $61 \%$, nonexclusively. This very substantial and significant increase of breastfeeding rates at hospital discharge confirmed the positive effect of the support offered to mothers. The effects of providing support to mothers, by the presence of doulas or specialized professionals, have been known to improve breastfeeding rates for a long time. ${ }^{15,25-27}$ This study confirmed those previous findings for mothers of preterm infants in a prospective randomized study. The breastfeeding rates at hospital discharge found after the intervention were similar to those reported for services with the best rates. ${ }^{10}$

The progression of breastfeeding at outpatient follow-up also provided important information that is rarely available in the literature. Median breastfeeding duration in the group of infants that were being breastfed at hospital discharge, excluding, therefore, those that were not breastfed, was 54 days in the routine group and 91 days in the intervention day $(p<0.01)$. Once more, the data of the routine group were similar to those previously found in studies conducted in our Service. ${ }^{9,19}$ The weaning rate was greater in the routine group, and the difference from the intervention group was observed up to the third month of outpatient follow-up of premature infants.

Despite the improvement of breastfeeding rates in the intervention group during all outpatient follow-up, the analysis of the survival curves showed that the rate of weaning during outpatient follow-up, indicated by the curve slope, was similar in the two groups (Figure 1). Similarly to other findings in the literature, ${ }^{28}$ the intervention during outpatient follow-up was not enough to reduce weaning rates.
Another important aspect, the effect of type of breastfeeding at hospital discharge, may be observed in Figure 2. The weaning rate of infants that were being exclusively breastfed at hospital discharge was lower than that of children receiving nonexclusive breastfeeding, regardless of study group. Exclusive breastfeeding has been described as an important factor in the continuation of breastfeeding in preterm infants. ${ }^{29}$

Some of the limitations of this study should be discussed. Although randomized, this study was not blinded, that is, data about the two groups were recorded and reviewed by the researcher. Despite the significant exclusion of motherinfant dyads recruited for the study, the sample size was large enough to detect the beneficial effects of the intervention. The number of children being exclusively breastfed at hospital discharge in each study group was small, which precluded the separate analysis of this variable.

In conclusion, this study demonstrated the importance of support and encouragement offered to the mother during the preterm infant's hospital stay. Simple measures, such as presence, support and frequent information from the researcher, had clear effects on breastfeeding rates at hospital discharge. Nonexclusive breastfeeding, which had a high incidence in this study, may indicate a failure in effectively establishing milk production, or in creating a good motherinfant bond. Therefore, future studies should improve the efficacy of this model and focus efforts on effective and exclusive breastfeeding at hospital discharge.

\section{References}

1. Wolff $P$. The serial organization of sucking in the young infant. Pediatrics. 1968;42:943-56.

2. Gryboski JD. Suck and swallow in the premature infant. Pediatrics. 1969;43:96-102.

3. Barnett CR, Leiderman PH, Grobstein R, Klaus M. Neonatal separation: the maternal side of interactional deprivation. Pediatrics. 1970;45:197-205.

4. Linhares MBM, Carvalho AEV, Bordin MBM, Chimello JT, Martinez $\mathrm{FE}$, Jorge SM.Prematuridade e muito baixo peso como fatores de risco ao desenvolvimento da criança. Paidéia. 2000;10:60-9.

5. Carvalho AEV, Linhares MBM, Martinez FE. História de desenvolvimento e comportamento de crianças nascidas prétermo e baixo peso (< 1500 g). Psicol Reflex Crit. 2001;14:133.

6. Hawkins-Walsh E. Diminishing anxiety in parents of sick newborns. MCN Am J Matern Child Nurs. 1980;5:30-4.

7. Elander G, Lindberg T. Short mother-infant separation during first week of life influences the duration of breastfeeding. Acta Paediatr Scand. 1984;73:237-40.

8. Procianoy RS, Fernandes Filho PH, Lazaro L, Sartori N. Influência de fatores neonatais sobre o aleitamento materno. J Pediatr (Rio J). 1982; 53:327-9. 
9. Xavier CC, Jorge SM, Gonçalves AL. Prevalência do aleitamento materno em recém-nascidos de baixo peso. Rev Saude Publica. $1991 ; 25: 381-7$.

10. Nascimento MB, Issler $\mathrm{H}$. Aleitamento materno em prematuros: manejo clínico hospitalar. J Pediatr (Rio J). 2004;80(5 Supl):S163-72.

11. McCoy R, Kadowaki C, Wilks S, Engstrom J, Meier P. Nursing management of breast feeding for preterm infants. J Perinat Neonatal Nurs. 1988;2:42-55.

12. Gonzalez KA, Meinzen-Derr J, Burke BL, Hibler AJ, Kavinsky B, Hess $S$, et al. Evaluation of a lactation support service in a children's hospital neonatal intensive care unit. J Hum Lact. 2003;19:286-92.

13. Sisk PM, Lovelady CA, Dillard RG, Gruber KJ. Lactation counseling for mothers of very low birth weight infants: effect on maternal anxiety and infant intake of human milk. Pediatrics. 2006; 117:e67-75.

14. Castrucci BC, Hoover KL, Lim S, Maus KC. Availability of lactation counseling services influences breastfeeding among infants admitted to neonatal intensive care units. Am J Health Promot. 2007;21:410-5.

15. Klaus $\mathrm{MH}$, Kennel $\mathrm{JH}$. The doula: an essential ingredient of childbirth rediscovered. Acta Paediatr. 1997;86:1034-6.

16. Langer A, Campero L, Garcia C, Reynoso S. Effects of psychosocial support during labour and childbirth on breastfeeding, medical interventions, and mothers' wellbeing in a Mexican public hospital: a randomised clinical trial. $\mathrm{Br}$ J Obstet Gynaecol. 1998;105:1056-63.

17. Merewood A, Philipp BL. Peer counselors for breastfeeding mothers in the hospital settings: trials, training, tributes and tribulations. J Hum Lact. 2003;19:72-6.

18. Scott JA, Landers MC, Hughes RM, Binns CW. Psychosocial factors associated with the abandonment of breastfeeding prior to hospital discharge. J Hum Lact. 2001;17:24-30.

19. Rocha NM, Martinez FE, Jorge SM. Cup or bottle for preterm infants: effects on oxygen saturation, weight gain, and breastfeeding. J Hum Lact. 2002;18:132-8.

20. Sweet L. Breastfeeding a preterm infant and the objectification of breastmilk. Breastfeed Rev. 2006;14:5-13.
21. Weimers L, Svensson K, Dumas L, Naver L, Wahlberg V. Hands-on approach during breastfeeding support in a neonatal intensive care unit: a qualitative study of Swedish mothers' experiences. Int Breastfeed J. 2006;26:1-20.

22. Pinelli J, Atkinson SA, Saigal S. Randomized trial of breastfeeding support in very low-birth-weight infants. Arch Pediatr Adolesc Med. 2001;155:548-53.

23. de Oliveira LD, Giugliani ER, do Espírito Santo LC, França MC, Weigert EM, Kohler CV, et al. Effect of intervention to improve breastfeeding technique on the frequency of exclusive breastfeeding and lactation-related problems. J Hum Lact. 2006; 22:315-21.

24. Fewtrell MS, Loh KL, Blake A, Ridout DA, Hawdon J. Randomized, double blind trial of oxytocin nasal spray in mothers expressing breast milk for preterm infants. Arch Dis Child Fetal Neonatal Ed. 2006;91:F169-74.

25. McCoy R, Kadowaki C, Wilks S, Engstrom J, Meier P. Nursing management of breastfeeding for preterm infants. J Perinat Neonatal Nurs. $1988 ; 2: 42-55$.

26. Memmott MM, Bonuck KA. Mother's reactions to a skills-based breastfeeding promotion intervention. Matern Child Nutr. 2006; 2:40-50.

27. Hill PD, Aldag JC, Chatterton RT, Zinaman M. Comparison of milk output between mothers of preterm and term infants: the first 6 weeks after birth. J Hum Lact. 2005;21:22-30.

28. Pinelli J, Atkinson SA, Saigal S. Randomized trial of breastfeeding support in very low-birth-weight infants. Arch Pediatr Adolesc Med. 2001;155:548-53.

29. Conde-Agudelo A, Diaz-Rossello JL, Belizan JM. Kangaroo mother care to reduce morbidity and mortality in low birthweight infants. Cochrane Database Syst Rev. 2003;(2):CD002771.

Correspondence:

Francisco Eulógio Martinez

Departamento de Puericultura e Pediatria da Faculdade de

Medicina de Ribeirão Preto, USP

Av. Bandeirantes, 3900

CEP 14049-900 - Ribeirão Preto, SP - Brazil

Tel.: + 55 (16) 3602.2573

Fax: +55 (16) 3602.2700

E-mail: femartin@fmrp.usp.br 